\title{
State-of-the-art Microanalysis at the nm-scale and Smaller: Going from Pretty Pictures to Quantitative Analysis of Hyperspectral Data
}

Paul G. Kotula

Sandia National Laboratories, PO Box 5800, Albuquerque, NM 87185-0886 USA

paul.kotula@sandia.gov

Analytical electron microscopy is currently undergoing a renaissance with the advent of aberration correction [1] and large solid-angle silicon-drift energy-dispersive x-ray spectrometers [2]. It is now easier than ever to collect large hyperspectral $\mathrm{x}$-ray data but is still important to have a sensible data acquisition and analysis strategy [3]. This tutorial will provide a general background on how to set up and acquire an appropriate amount of data allowing subsequent qualitative and quantitative analyses. It will also describe robust multivariate statistical analysis approaches and methods for quantification of such data sets and examples from a number of material's systems.

Regardless of instrumentation used (newer versus older systems) there are some general rules of thumb for acquiring data with counts to perform conventional or more sophisticated analyses. It is important to aim for between 100 and 300 counts in each spectrum [4]. This can be achieved by first moving the probe around the specimen to determine the counts per second output from the EDS system and then setting an appropriate per pixel total dwell time. For example, if we have $3000 \mathrm{cps}$ from a specimen then 100 msec total per pixel dwell time would give us 300 counts per spectrum on average. Regardless of whether the goal is qualitative or quantitative analysis, the signal range or 100-300 counts per pixel should be considered in setting up an experiment. If the goal is higher analytical sensitivity then higher counts per spectrum should be considered. In the example below, the average counts per pixel (spectrum) were close to 1000 .

An example of this the process of collecting and analyzing spectral image data will be given for the Carlton iron meteorite which consists, in the region of interest, of a low $\mathrm{Ni}$ phase (Kamacite), a high Ni phase (clear Taenite) and then at lower Ni concentrations a spinodal which is a fine two-phase region as shown in Fig. 1A. A Spectral image was acquired with an FEI Company Tecnai F-30ST STEM operating at 300kV with a probe size of $\sim 2 \mathrm{~nm}$ and a probe current of $\sim 2 \mathrm{nA}$ and equipped with an EDAX r-TEM EDS system. The FIB specimen gave $\sim 3000$ counts per second from this region and paired with a 300 msec per-pixel dwell time provided 1000 counts per pixel in the final data. A narrow spectral region comprising the Fe and Ni K-peaks was analyzed with Sandia's multivariate statistical analysis software [4-5] to separate the Fe and Ni signals from each other as shown in Figs 1B-D. These component images in Figs $1 \mathrm{~B}$ and C consist of counts for the respective elements and can be quantified with the Cliff-Lorimer approach after measuring $\mathrm{k}_{\mathrm{NiFe}}=1.10$ from a standard specimen with the resulting quantified images shown in Figs. 1E and $\mathrm{F}$. The arrows point to compositional variations of about 0.75 wt. $\%$ in the clear Taenite layer. 
[1] H.Müller, S.Uhlemann,P.Hartel,M.Haider, Microsc. Microanal.12 (2006) 442.

[2] Von Harrach, et al. J Phys 241, 012015 (2010).

[3] P.G. Kotula, et al. Microsc. Micoranal. 18 691-698 (2012)

[4] P.G. Kotula, M.R. Keenan, \& J.R. Michael. Microsc. Microanal. 9 [1] 1-17 (2003).

[5] M.R. Keenan \& P.G. Kotula. Surf. Int. Anal. 36 203-212 (2004).

Prof. Joseph Goldstein is acknowledged for provide the meteorite specimen analyzed in this work. Sandia is a multiprogram laboratory operated by Sandia Corporation, a Lockheed Martin Company, for the United Stated Department of Energy's (DOE) National Nuclear Security Administration (NNSA) under contract DE-AC0494AL85000.
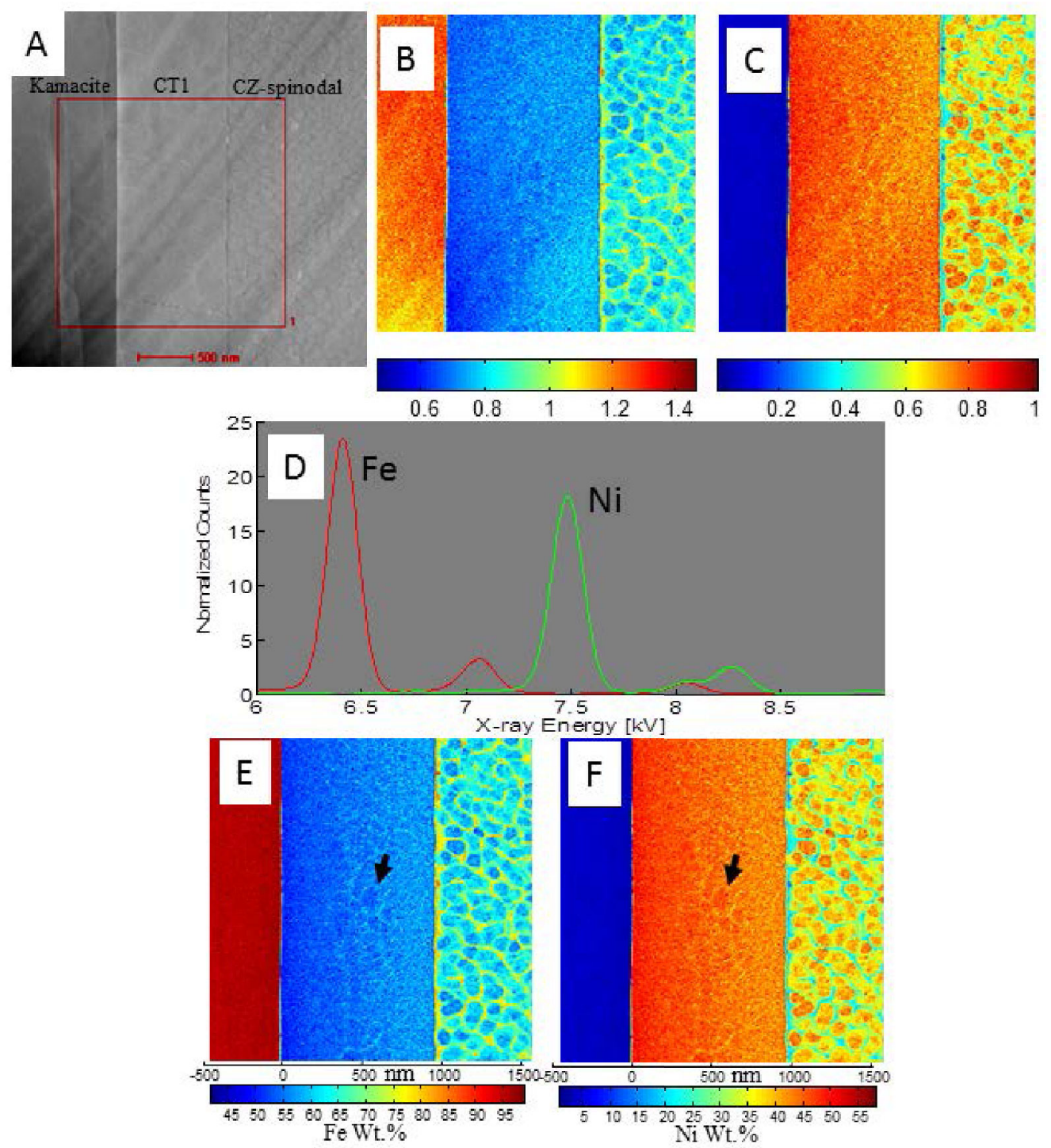

Figure 1. A. HAADF-STEM image taken from the region of interest at the KamaciteTaenite phase boundary. B, C, and D. Component images of $\mathrm{Fe}, \mathrm{Ni}$ and the respective spectral shapes from the analysis of the spectral image. E, F. Quantified spectral image data for $\mathrm{Fe}$ and $\mathrm{Ni}$ in weight percent with arrows indicating subtle composition variations in the single-phase clear Taenite region. 